\title{
High-definition ultrasound characterization of acute cyclophosphamide-induced cystitis in the mouse
}

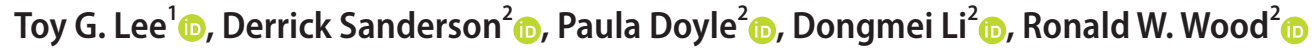 \\ 'Department of Obstetrics and Gynecology, University of Texas Medical Branch, TX, ${ }^{2}$ Department of Obstetrics and Gynecology, University of Rochester Medical Center, \\ NY, USA
}

Purpose: To examine associations if any between changes in voiding function, hematuria, and bladder ultrasonography metrics in murine cyclophosphamide-induced chemical cystitis.

Materials and Methods: Cystitis was induced in 6 female mice by an intraperitoneal injection of cyclophosphamide (300 mg/ $\mathrm{kg}$ ). Voiding frequency, void volume, hematuria assessment, and ultrasonographic measurements of the bladder were obtained at baseline, days 1 to 5, and days 9, 11, and 13. Voiding was induced with preferred sweet drinking solution and voiding data collected using an automated data collection system in 135 minute sessions. Bladder wall thickness, lumen volume, and vascular Doppler were acquired using a high definition ultrasound system. Spearman's correlation was used to analyze the association between the voiding changes, hematuria, and ultrasound findings.

Results: Hematuria was present 24 hours after cyclophosphamide injection. All animals displayed increased bladder vascularity, bladder wall thickness, and void frequency that was associated with concurrent decreased total and average void volumes. Increased bladder wall vascularity was correlated with the presence of hematuria $(r=0.59, p<0.01)$ and bladder wall thickness $(r=0.79$, $p<0.01)$. Hematuria correlated with increased void frequency $(r=0.34, p<0.01)$. Average void volume was negatively correlated with hematuria $(r=-0.50, p<0.01)$ and frequency $(r=-0.38, p<0.01)$.

Conclusions: High-definition ultrasound imaging permits in vivo monitoring of changes in bladder morphology associated with voiding function in relation to cyclophosphamide-induced cystitis. Ultrasound imaging of the bladder may assist in differential diagnosis of bladder dysfunction.

Keywords: Animals; Cyclophosphamide; Cystitis; Ultrasonography; Urinary bladder

This is an Open Access article distributed under the terms of the Creative Commons Attribution Non-Commercial License (http://creativecommons.org/licenses/by-nc/4.0) which permits unrestricted non-commercial use, distribution, and reproduction in any medium, provided the original work is properly cited.

\section{INTRODUCTION}

Approximately $6.5 \%$ of adult women in the United States (US) are affected by interstitial cystitis (IC) [1], a condition characterized by pain, pressure, or discomfort attributed to the bladder and is often associated with urinary urgency and frequency [2]. Symptoms are variable and patient dependent but quality of life can be severely impacted by anxiety,

Received: 11 June, 2019 - Accepted: 1 September, 2019

Corresponding Author: Toy G. Lee (iD https://orcid.org/0000-0003-2063-6033

Department of Obstetrics and Gynecology, University of Texas Medical Branch, 301 University Blvd, 335 Clinical Science Building, Galveston, Texas 77550-

0587, USA

TEL: +1-713-855-8572, FAX: +1-585-276-1956, E-mail: tolee@utmb.edu 
sexual dysfunction, sleep disturbance, and impaired work productivity [3] Symptoms may persist despite treatment.

The etiology of IC is unknown, hence preclinical investigations typically rely on inducing voiding dysfunction, typically with a chemical challenge to the bladder. Cyclophosphamide (CYP) reliably induces a chemical cystitis that is useful in the context of preclinical IC research [4-6]. CYP is a stable nitrogen mustard that selectively activates in phosphatase and phosphamidase rich cellular environments [7]. CYP is hydrolyzed to acrolein in the bladder causing urothelial injury, resulting in hematuria and changes in void volume and frequency [5,6]. Damage to the urinary tract after CYP injection was early recognized as a potential adverse effect and is readily prevented [8]

Despite innovations leading to automation and non-invasive techniques for monitoring voiding function [9-13], there has, to date, been no attempt to use non-invasive imaging modalities such as high resolution ultrasound to characterize changes in bladder dimension or blood flow after induction of cystitis from CYP injury. Noninvasive ultrasonography offers the promise of being a reliable tool for in vivo examination of the bladder and might be translated to an additional tool for differential diagnosis of bladder dysfunction and pelvic pain. The objective of this study is to evaluate whether high definition ultrasound is able to detect bladder morphological changes caused by acute CYP injury.

\section{MATERIALS AND METHODS}

After receiving approval from the University Committee of Animal Research (UCAR) (approval number: UCAR 2006-083R) and the University of Rochester Medical Center (Rochester, NY, USA), six 10 to 12 week-old female CRL (Charles River Lab, Boston, MA, USA) CD-1 mice were obtained. The mice were housed 3 mice per polycarbonate cages. The animals were housed in these home cages in a reverse light cycle room with unrestricted access to food and water. The mice were offered a sweet solution ( $3 \%$ dextrose and $0.125 \%$ saccharin) during the dark phase of the reverse light cycle that reliably induces polydipsia [14] and enhances the urine output; 6 milliliters of this solution were placed in a drinking tube attached to the diuresis cage before each session.

\section{Voiding sessions}

Voiding sessions were performed at baseline for 3 days and on post-injection days 1 to 5, 9, 11, and 13. Each session lasted 135-minutes and were performed using an automated, noninvasive voiding system [9]. Each voiding cage utilized metabolic cage tops with rat floor grids (Tecniplast, Buguggiate, Italy) positioned over $1 \mathrm{mg}$ resolution electronic balances (Professional Series, Mettler-Toledo, Columbus, OH, USA). Additionally, each animal was recorded with a highdefinition camera throughout each voiding session (Fig. 1).

During the voiding sessions mice had access to $6 \mathrm{~mL}$ of the saccharin solution described above in a double ball bearing sipper tube in the diuresis cages; no solid food was available during the voiding sessions to prevent erroneous events in the automated data collection.

After previous experimental work identified the performance criteria for an optimal voiding function evaluation, a new software system was written (Robert H. Schor, PhD) and used for the first time in this series of experiments and is described briefly here. Balances reported and their status recorded at 10 times per second; cameras streamed images at 30 frames per second. A video file was created every time the scale noted a weight increase of $5 \mathrm{mg}$ that included camera images from 2 seconds before the event and ending when there were no changes greater than $3 \mathrm{mg}$ for 2 seconds. Each event was later reviewed by the lead author (TL); fecal events were excluded, thus only voiding events were included in the data analysis.

Hematuria in urine on the plate below the mouse was determined immediately after the conclusion of the $135 \mathrm{~min}$ ute voiding session using standard urine dipsticks (Chemstrip 7; Roche Diagnostics, Indianapolis, IN, USA). Hematuria was recorded as negative, $1+, 2+$, or $3+$.

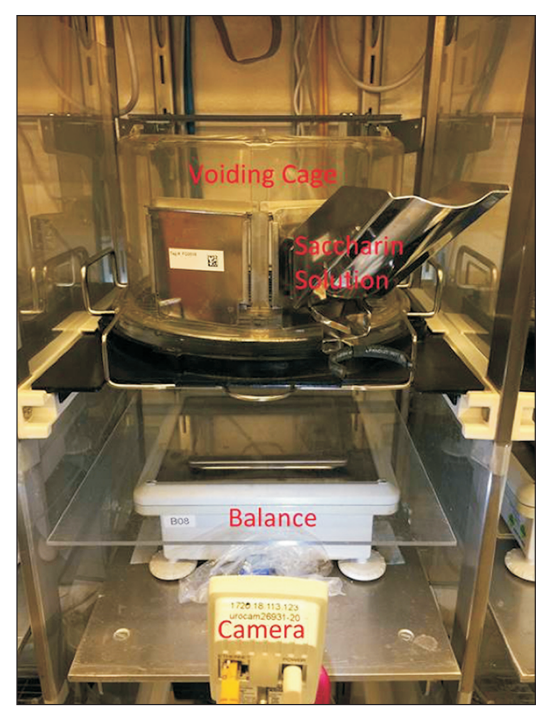

Fig. 1. Voiding cage. Metabolic cage and rat grid floor with access to saccharin solution, above sensitive balance. Session recording by high definition camera. 


\section{Cystitis induction protocol}

After collection of baseline voiding data, CYP (300 mg/ $\mathrm{kg}$ ) was administered intraperitoneally. This dose of CYP is nonlethal [15] and reliably produces changes in voiding frequency, void volume, and hematuria [9].

\section{Ultrasound imaging}

Ultrasound imaging was performed following the voiding sessions at baseline and on days 1 to $5,9,11$, and 13. Isoflurane ( $2 \%$ in oxygen) was administered via a snout tube during the ultrasound sessions. Each mouse was comfortably secured to a warm temperature-controlled platform with integrated physiologic monitoring capabilities throughout each imaging session. The VEVO 3100 high resolution ultrasound system (Visual Sonics, Toronto, ON, Canada) with the MX550D transducer probe was used with settings optimized for small animal abdominal imaging, including respiratory gating to eliminate motion artifacts. Three dimensional Bmode and power Doppler images were obtained sequentially with an axial resolution of 40 microns. Fig. 2 highlights samples of grayscale B-mode and Doppler images from a representative animal from select days of the study. All images were acquired during expiratory phase of respiration (image gating). The mice completely recovered from anesthesia before being returned to their home cages in the reverse light cycle room.

\section{Image analysis}

Amira ${ }^{\mathrm{TM}}$ FEI systems (Thermo Fisher Scientific, Hillsboro, OR, USA) was used for segmentation, quantification, and display of these data. Bladder wall and lumen volume were obtained by segmenting the border wall with the interpolation feature after using an edge-preserving bilateral digital filter. The Doppler flow volume of the area inside the bladder wall was obtained by masking the 3D Doppler array by the segmented bladder tissue excluding the bladder lumen.

\section{Statistical analysis}

Summary statistics were used to describe voiding changes, hematuria, and ultrasound findings for each individual animal and across all animals. Spearman's correlation coefficients were used to quantify associations between changes in void frequency, void volume, hematuria, and ultrasound metrics. All analyses were conducted using Statistical Analysis Software SAS ver. 9.4 (SAS Institute Inc., Cary, NC, USA) with $\mathrm{p}<0.05$ considered statistically significant.

\section{RESULTS}

All of the mice $(n=6)$ exhibited changes in voiding function and bladder morphology with hematuria present 24 hours following CYP injection (Table 1). The number of animals with hematuria decreased each day of the experiment and had resolved by day 5 .

Table 1 also illustrates the median changes (expressed as \%) from baseline for each experimental day for voiding function and sonographic changes. The animals demonstrated an increase in voiding frequency (243\%) with a corresponding decrease in average void volume $(-71 \%)$ and total void volume (-14\%) on day 1 . Ultrasonographic findings on day 1 demonstrated an increase in bladder wall thickness

\section{Baseline}
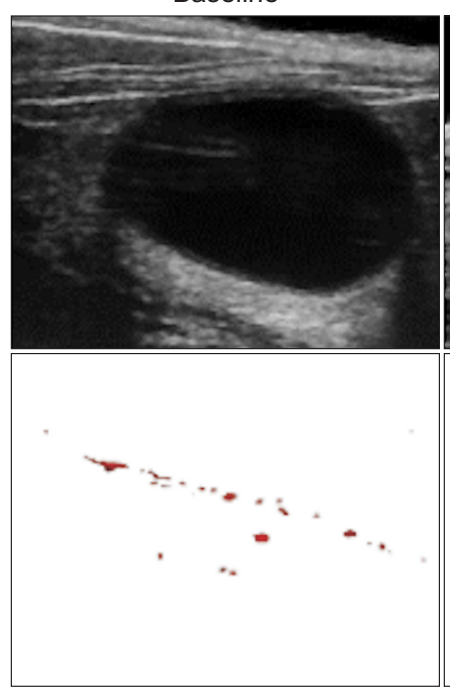

Day 1

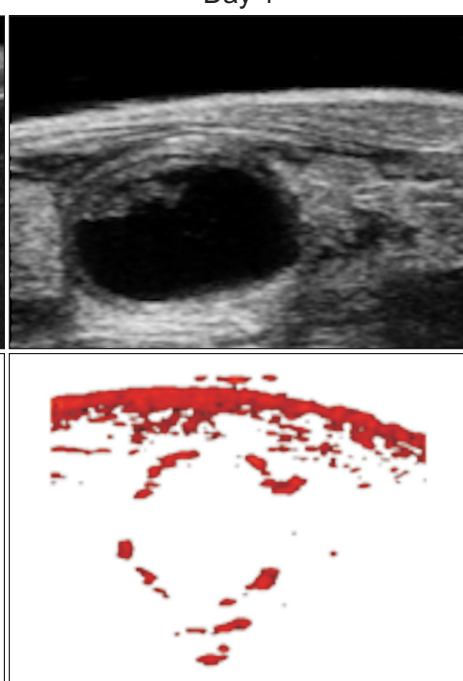

Day 3

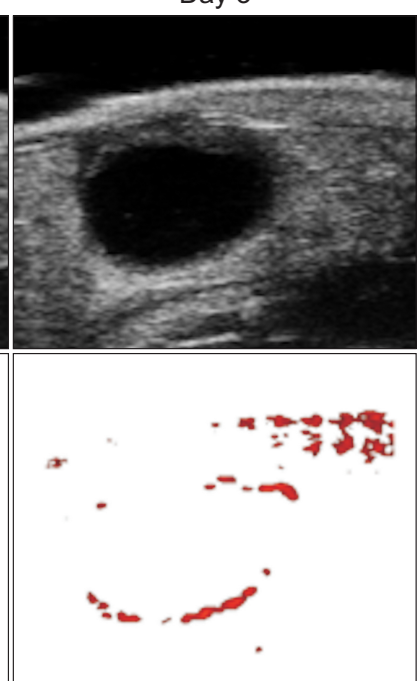

Day 13

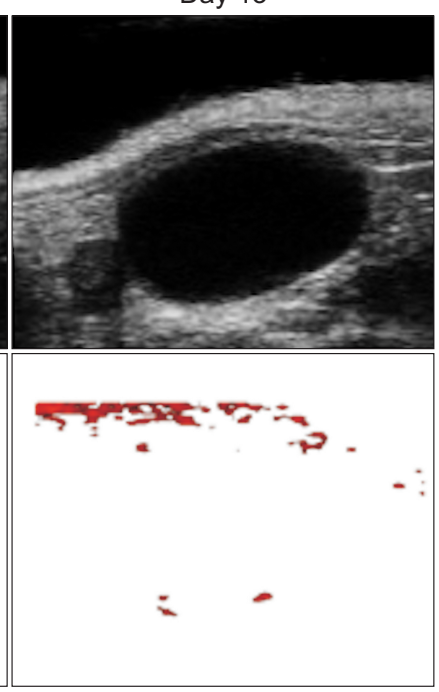

Fig. 2. Ultrasound images. B-mode and Doppler ultrasound images from baseline, day 1, day 3, and day 13 from a representative mouse. 
(154\%) and bladder wall vascularity (53,586\%) with concomitant decreased bladder lumen (-66\%).

Despite the resolution of hematuria, functional and morphologic changes persisted with only total void volume and bladder wall thickness returning to within $\leq 5 \%$ of baseline by day 13. The resolution of hematuria does not correspond to full recovery of average void volume. Bladder lumen volume and bladder wall vascularity also did not demonstrate a return to baseline measurements.

Table 2 displays results from the Spearman's correlation analysis. Positive correlations were found between increased bladder vascularity and increasing hematuria $(r=0.59, p<0.01)$ as well as increased bladder wall thickness $(r=0.79, p<0.01)$ (Table 2). Increased vascularity was weakly correlated with voiding frequency $(r=0.17, p<0.01)$ despite the strong relationship with decreased average void volumes $(r=0.55, \mathrm{p}<0.01)$. Increasing hematuria was also positively correlated with voiding frequency $(\mathrm{r}=0.34, \mathrm{p}<0.01)$ and bladder wall thickness $(r=0.41, p<0.01)$ while being negatively correlated with average void volume $(r=0.50, p<0.01)$ and lumen volume $(r=-$ 0.19, $\mathrm{p}<0.01$ ). Surprisingly, an association was not apparent between the volumetric changes of the bladder lumen and the mean void volume changes $(r=-0.00078, p=0.980)$ following CYP induced cystitis.

\section{DISCUSSION}

This is the first experiment to investigate and establish the utility of noninvasive, in vivo high definition ultrasound imaging in murine chemical cystitis. Our results demonstrate that high-definition ultrasound with power Doppler can measure changes in bladder morphology and vascularity during cystitis without untoward effects in the animal while detecting significant increases in bladder wall vascularity resulting from CYP-induced cystitis. Acute changes of urinary frequency and void volumes occurred within 24 hours after cystitis induction consistent with previous experimental findings $[9,16]$. Hematuria was associated with decreased functional bladder capacity $(\mathrm{r}=-0.50, \mathrm{p}<0.01)$, decreased bladder lumen size $(r=0.19, \mathrm{p}<0.001)$, increased voiding frequency $(r=0.34, p<0.01)$, increased bladder wall thickness $(r=0.41, p<0.01)$, and increased bladder wall vascularity $(\mathrm{r}=0.59, \mathrm{p}<0.01)$. However, we discovered that resolution of hematuria did not correlate with a full resolution of voiding symptoms. Increased vascularity was associated with more frequent urination $(r=0.17, p<0.01)$ and smaller void volumes $(\mathrm{r}=0.55, \mathrm{p}<0.01)$. Doppler ultrasonography and the reconstruction algorithms documented an unanticipated enormous increase in the median bladder wall vascularity during CYP

Table 1. Voiding function and ultrasonographic changes

\begin{tabular}{|c|c|c|c|c|c|c|c|}
\hline Day & $\begin{array}{c}\text { Median change } \\
\text { in void } \\
\text { frequency }\end{array}$ & $\begin{array}{c}\text { Median change } \\
\text { in average void } \\
\text { volume }\end{array}$ & $\begin{array}{l}\text { Median change } \\
\text { in total void } \\
\text { volume }\end{array}$ & $\begin{array}{c}\text { Median change } \\
\text { in bladder wall } \\
\text { thickness }\end{array}$ & $\begin{array}{c}\text { Median change } \\
\text { in bladder lumen } \\
\text { volume }\end{array}$ & $\begin{array}{c}\text { Median change } \\
\text { in bladder wall } \\
\text { vascularity }\end{array}$ & $\begin{array}{c}\text { Animals with } \\
\text { hematuria }\end{array}$ \\
\hline 1 & $243(153)$ & $-71(12)$ & $-14(26)$ & $154(289)$ & $-66(76)$ & $53,586(125,790)$ & 6 \\
\hline 2 & $92(233)$ & $-45(48)$ & $16(42)$ & $128(127)$ & $-41(55)$ & $20,450(137,342)$ & 4 \\
\hline 3 & $36(58)$ & $-25(36)$ & $8.4(62)$ & $86(163)$ & $-23(67)$ & $28,400(170,245)$ & 3 \\
\hline 4 & 24 (149) & $-28(40)$ & $-0.07(55)$ & $110(115)$ & $-14(82)$ & $37,900(75,379)$ & 1 \\
\hline 5 & $-40(40)$ & $-29(58)$ & $-55(56)$ & $136(221)$ & $14(137)$ & $4,900(35,390)$ & 0 \\
\hline 9 & $6.5(274)$ & $-44(77)$ & $-35(44)$ & $49(50)$ & $-35(50)$ & $4,990(38,766)$ & 0 \\
\hline 11 & $130(292)$ & $-34(68)$ & $23(50)$ & $12(86)$ & $-39(56)$ & $1,685(11,511)$ & 0 \\
\hline 13 & $27(128)$ & $-45(70)$ & $5(102)$ & $-3(65)$ & $-46(50)$ & $2,809(9,237)$ & 0 \\
\hline
\end{tabular}

Values are presented as percent median change from baseline (interquartile range) or number only. Voiding session, ultrasound data, and hematuria for days 1 to $5,9,11$, and 13 post-cyclophosphamide injection.

Table 2. Spearman's correlation analysis

\begin{tabular}{lcccccc} 
& Hematuria & Frequency & Average void & Doppler & Lumen & Bladder wall \\
\hline Hematuria & 1 & 0.34 & -0.50 & 0.59 & -0.19 & 0.41 \\
Frequency & & 1 & -0.38 & 0.17 & 0.16 & -0.13 \\
Average void & & & 1 & -0.55 & $-0.00078\left(p=0.980^{\mathrm{a}}\right)$ & 0.12 \\
Doppler & & & & 1 & 1 & 0.79 \\
Lumen & & & & & 1 \\
Bladder wall & & & & & & 0.46 \\
\hline
\end{tabular}

Data are Spearman's correlation coefficient ( $r$ ).

a:All were statistically significant with $p<0.01$ with the exception of this coefficient. 
induced cystitis. This decreased over the study period but vascularity never returned to baseline.

Limitations of our experiment include the small experimental cohort $(n=6)$ and lack of histology. This was an initial pilot study to investigate the possibility of in vivo, high definition ultrasound as an imaging modality to noninvasively identify morphologic changes in the mouse bladder and an association, if any, with changes in voiding function. Our objective was to noninvasively image the impact, followed by resolution of injury in the same animal to investigate the sensitivity of ultrasound measurements and Doppler on assessing these changes. On day 1 , we noted a significant and clearly visible $\sim 500$ times increase in vascularity in our ultrasound imaging. However, this was not confirmed with histology. We hope to address this limitation with in future studies with larger animal groups while performing histology at different time points to correlate with ultrasound data. We also sought to establish techniques to study vascularity and inflammation with Doppler measurements. A difficulty during the experiment was the inability to control for bladder volumes during imaging, leading to potential error in using ultrasound imaging for quantification of bladder characteristics. The authors are currently developing an improved backfill method using a small gauge angiocatheter that minimizes any possible bladder trauma, which would standardize bladder volumes during imaging.

Due to the short term effects of a single dose of CYP, this study is a better reflection of acute inflammatory bladder changes. A longer study period with repeated doses of CYP may be necessary to provide important information regarding the duration of morphologic changes due to chronic CYP induced cystitis, and therefore, more accurately mimic the chronic changes seen in those with IC [17,18].

Bladder wall edema and decreased bladder lumen were clearly observed on ultrasound imaging which is consistent with previous findings from immunohistological evaluation [19]. This is similar to clinical studies that have noted increased bladder wall thickness in detrusor overactivity [20] and IC [17]. Bladder wall thickness is associated with decreased bladder capacity and worsened symptom severity in human subjects [17] However, there is a paucity of literature related to cystitis induced vascular changes in the setting of benign voiding dysfunction. Pontari et al. [18] noted decreased blood flow in those with IC compared to those without IC by focal laser. Further pre-clinical and clinical imaging research is needed to examine the reliability of in vivo imaging in changes associated with IC and IC models. This study highlights the translational promise of using ultrasound with Doppler capability to characterize bladder morphology and vascularity in patients with and without IC and other lower urinary tract symptoms such as overactive bladder. Differences in bladder edema, lumen size, and bladder vascularity in symptomatic patients may potentially provide a noninvasive means of characterizing the severity of their disease state and provide guidance towards treatment options.

\section{CONCLUSIONS}

In conclusion, high definition ultrasound with power Doppler can be a used to observe in vivo changes in bladder morphology related to cystitis without the need for invasive procedures or ionizing radiation providing a low-cost, high throughput platform for pre-clinical assessment. The voiding collection software developed for this experiment can be used for future murine voiding models. Continued investigation will be necessary to fully elucidate the role and reliability of clinical ultrasound imaging in IC.

\section{CONFLICTS OF INTEREST}

The authors have nothing to disclose.

\section{ACKNOWLEDGMENTS}

Ronald W. Wood, Robert H. Schor, and Mae Stone Goode Grant Program GR500735.

\section{AUTHORS' CONTRIBUTIONS}

Research conception and design: Toy G. Lee and Ronald W. Wood. Data acquisition: Toy G. Lee. Statistical analysis: Dongmei Li. Data analysis and interpretation: Toy G. Lee, Derrick Sanderson, Paula Doyle, Dongmei Li, and Ronald W. Wood. Drafting of the manuscript: Toy G. Lee, Derrick Sanderson, Paula Doyle, Dongmei Li, and Ronald W. Wood. Critical revision of the manuscript: Toy G. Lee, Derrick Sanderson, Paula Doyle, and Ronald W. Wood. Obtaining funding: Toy G. Lee. Administrative, technical, or material support: Ronald W. Wood. Supervision: Ronald W. Wood. Approval of the final manuscript: Toy G. Lee, Derrick Sanderson, Paula Doyle, and Ronald W. Wood.

\section{REFERENCES}

1. Berry SH, Elliott MN, Suttorp M, Bogart LM, Stoto MA, Eggers $\mathrm{P}$, et al. Prevalence of symptoms of bladder pain syndrome/ interstitial cystitis among adult females in the United States. J 
Urol 2011;186:540-4.

2. Hanno PM, Burks DA, Clemens JQ, Dmochowski RR, Erickson D, Fitzgerald MP, et al.; Interstitial Cystitis Guidelines Panel of the American Urological Association Education and Research, Inc. AUA guideline for the diagnosis and treatment of interstitial cystitis/bladder pain syndrome. J Urol 2011;185:2162-70.

3. Vasudevan V, Moldwin R. Addressing quality of life in the patient with interstitial cystitis/bladder pain syndrome. Asian J Urol 2017;4:50-4.

4. Boucher M, Meen M, Codron JP, Coudore F, Kemeny JL, Eschalier A. Cyclophosphamide-induced cystitis in freelymoving conscious rats: behavioral approach to a new model of visceral pain. J Urol 2000;164:203-8.

5. Edrees G, Luts A, Stewart F. Bladder damage in mice after combined treatment with cyclophosphamide and X-rays. The influence of timing and sequence. Radiother Oncol 1988;11:349-60.

6. Stewart FA. Mechanism of bladder damage and repair after treatment with radiation and cytostatic drugs. Br J Cancer Suppl 1986;7:280-91.

7. Arnold H, Bourseaux F, Brock N. Chemotherapeutic action of a cyclic nitrogen mustard phosphamide ester (B 518-ASTA) in experimental tumours of the rat. Nature 1958;181:931.

8. Rubin JS, Rubin RT. Cyclophosphamide hemorrhagic cystitis. J Urol 1966;96:313-6.

9. Wood R, Eichel L, Messing EM, Schwarz E. Automated noninvasive measurement of cyclophosphamide-induced changes in murine voiding frequency and volume. J Urol 2001;165:653-9.

10. Wood RW, Baggs RB, Schwarz EM, Messing EM. Initial observations of reduced uroflow in transgenic adenocarcinoma of murine prostate. Urology 2006;67:1324-8.

11. Nicholson TM, Ricke EA, Marker PC, Miano JM, Mayer RD, Timms BG, et al. Testosterone and $17 \beta$-estradiol induce glan- dular prostatic growth, bladder outlet obstruction, and voiding dysfunction in male mice. Endocrinology 2012;153:5556-65.

12. Nicholson TM, Nguyen JL, Leverson GE, Taylor JA, Vom Saal FS, Wood RW, et al. Endocrine disruptor bisphenol A is implicated in urinary voiding dysfunction in male mice. Am J Physiol Renal Physiol 2018;315:F1208-16.

13. Nicholson TM, Moses MA, Uchtmann KS, Keil KP, Bjorling $\mathrm{DE}$, Vezina CM, et al. Estrogen receptor- $\alpha$ is a key mediator and therapeutic target for bladder complications of benign prostatic hyperplasia. J Urol 2015;193:722-9.

14. Valenstein ES, Cox VC, Kakolewski JW. Polydipsia elicited by the synergistic action of a saccharin and glucose solution. Science 1967;157:552-4.

15. Datta K, Chin A, Ahmed T, Qing WG, Powell KL, Simhambhatla $\mathrm{P}$, et al. Mixed effects of 2,6-dithiopurine against cyclophosphamide mediated bladder and lung toxicity in mice. Toxicology 1998;125:1-11.

16. Bjorling DE, Elkahwaji JE, Bushman W, Janda LM, Boldon K, Hopkins WJ, et al. Acute acrolein-induced cystitis in mice. BJU Int 2007;99:1523-9.

17. Wu SY, Jhang JF, Jiang YH, Kuo HC. Increased bladder wall thickness is associated with severe symptoms and reduced bladder capacity in patients with bladder pain syndrome. Urol Sci 2016;27:263-8.

18. Pontari MA, Hanno PM, Ruggieri MR. Comparison of bladder blood flow in patients with and without interstitial cystitis. J Urol 1999;162:330-4.

19. Golubeva AV, Zhdanov AV, Mallel G, Dinan TG, Cryan JF. The mouse cyclophosphamide model of bladder pain syndrome: tissue characterization, immune profiling, and relationship to metabotropic glutamate receptors. Physiol Rep 2014;2:e00260.

20. Ali MM, Ahmed AF, Khaled SM, Abozeid H, AbdelMagid ME. Accuracy of ultrasound-measured bladder wall thickness for the diagnosis of detrusor overactivity. Afr J Urol 2015;21:25-9. 